\title{
Reflets
}

Revue ontaroise d'intervention sociale et communautaire

\section{L'engagement de la Caisse populaire dans le développement de la communauté de Kapuskasing}

\section{Natalie Dupont}

Volume 5, numéro 1, printemps 1999

Pratiques et développement économique communautaire

URI : https://id.erudit.org/iderudit/026257ar

DOI : https://doi.org/10.7202/026257ar

Aller au sommaire du numéro

Éditeur(s)

Reflets : Revue ontaroise d'intervention sociale et communautaire

ISSN

1203-4576 (imprimé)

1712-8498 (numérique)

Découvrir la revue

Citer cet article

Dupont, N. (1999). L'engagement de la Caisse populaire dans le développement de la communauté de Kapuskasing. Reflets, 5(1), 168-175.

https://doi.org/10.7202/026257ar

Tous droits réservés (C) Reflets : Revue ontaroise d'intervention sociale et communautaire, 1999
Ce document est protégé par la loi sur le droit d'auteur. L'utilisation des services d'Érudit (y compris la reproduction) est assujettie à sa politique d'utilisation que vous pouvez consulter en ligne.

https://apropos.erudit.org/fr/usagers/politique-dutilisation/ 


\section{L'engagement de la Caisse populaire dans le développement de la communauté de Kapuskasing $^{1}$}

\section{Natalie Dupont}

Étudiante, maîtrise en sociologie, Université Laurentienne

Le mouvement coopératif fait aujourd'hui l'objet d'un débat passionné. Certaines auteures et certains auteurs s'entendent pour dire que l'avenir des coopératives est en déclin; le capital prenant de plus en plus d'importance dans notre société, elles devront adopter des stratégies semblables à celles qu'utilisent les banques (Élie 1997). D'autres affirment que les coopératives sont destinées soit à disparaître, soit à adhérer à l'univers capitaliste (Labrecque 1986). Enfin, d'autres individus insistent que seuls des nouveaux modèles et des nouveaux modes de fonctionnement sauront sauver le mouvement coopératif (Malo et Lévesque 1997).

Une chose est certaine, malgré le débat qui existe quant à l'orientation du mouvement coopératif, les auteurs s'entendent pour dire que les coopératives et les caisses populaires vivent présentement des transformations sociales engendrées, d'une part, par l'intensification de la crise économique du début des années 1980 et d'autre part, par l'affirmation du néolibéralisme. Dans ce contexte, l'État s'est retiré de nombreuses sphères de la société. Les entreprises et les coopératives devant se trouver des stratégies pour survivre dans ce contexte social et économique, d'autres formes d'organisations se créent en réponse au retrait de l'État.

Dans la logique sociale et économique actuelle, l'avenir des coopératives et des caisses populaires demeure au centre d'un 
débat. Leur objectif de départ qui se voulait d'offrir un sentiment d'appartenance aux francophones (Lafrenière 1987), peut s'avérer impossible à maintenir aujourd'hui. À vrai dire, leur mission de base ne correspond peut-être plus aux demandes du marché qui est fondé, plus que jamais, sur la compétition, la rivalité et la concurrence.

«Les coopératives et les caisses populaires dévient-elles de leur mission?»
Une question plutôt générale découle de ce débat. Les coopératives et les caisses populaires dévient-elles de leur mission? Pour répondre à cette question, nous avons choisi d'étudier le cas et la mission de la Caisse populaire de Kapuskasing. La mission de cette caisse, adoptée en juin 1989, se lit comme suit.

Notre mission est de faire en sorte que la Caisse populaire de Kapuskasing soit l'institution financière préférée de la population parce qu'elle est la meilleure de la région:

1. à cause de l'accueil chaleureux, de la compétence et du dynamisme de son personnel toujours heureux de répondre aux besoins grandissants des sociétaires, et

2. parce qu'elle est perçue comme contribuant le plus à sa communauté et qu'elle respecte son héritage historique et culturel. (Caisse populaire de Kapuskasing, 1997, document informatisé).

Notre recherche a cherché à savoir jusqu'à quel point la Caisse de Kapuskasing remplit cette mission. Plus particulièrement, elle s'est penchée sur une dimension de cette mission en tentant de découvrir jusqu'à quel point les associations communautaires qui bénéficient d'une aide financière de la caisse populaire perçoivent cette dernière comme étant l'institution qui s'engage face à sa communauté et y contribue le plus.

Pour ce faire, nous avons interviewé trente et une (31) personnes membres d'associations communautaires de la région de Kapuskasing. À l'aide d'un questionnaire, nous avons obtenu des informations générales sur l'association, sur la contribution de la Caisse, sur l'image que se font les associations à l'égard de la Caisse et enfin, sur les images positives et négatives qui, d'après les répondantes et les répondants, sont véhiculées dans la communauté de Kapuskasing. 


\section{La perception des associations à l'égard de la contribution de la Caisse}

De façon générale, les répondantes et répondants reçoivent de l'aide financière de la Caisse populaire dans le but de financer un projet particulier. La plupart des répondantes et répondants n'ont pas demandé de montant spécifique lorsqu'ils ont fait une demande d'aide financière. Les conditions de la contribution ont été laissées à la discrétion de la Caisse dans $51,6 \%$ des cas. Notons, à cet effet, que $64,5 \%$ des associations ont reçu des montants inférieurs à $1000 \$$. Enfin, la Caisse ne demande pas que les associations répondent à des exigences particulières afin de recevoir une subvention; elle n'exige pas non plus qu'elles remettent un rapport d'activités pour justifier leur contribution.

Malgré le portrait que nous avons obtenu sur la perception des répondantes et répondants à l'égard de la contribution de la Caisse, les données présentées ci-dessous ne permettent pas de dégager leur satisfaction quant à la contribution. Quelles étaient leurs attentes de financement par rapport à la contribution de la Caisse? Quel est leur degré de satisfaction? Les associations fontelles appel à d'autres sources de financement et obtiennent-elles d'autre aide de la part de la Caisse à part la subvention?

\section{Satisfaction des répondantes et répondants quant à la contribution de la Caisse}

Les répondantes et répondants expriment presque unanimement que la contribution de la Caisse est «très satisfaisante». Les répondantes et répondants ont clairement indiqué que leurs attentes de financement sont assez élevées. La contribution de la Caisse populaire de Kapuskasing semble donc combler les besoins des projets des associations communautaires. D'autant plus que la 
Caisse procure d'autre aide en plus de la subvention. En effet, $59 \%$ des répondantes et des répondants ont mentionné une aide additionnelle telle «le maintien du parc» ou «la consultation au plan financier». Un répondant a même souligné l'aide inestimable fournie par le Directeur des communications à la Caisse.

Malgré ce niveau élevé de satisfaction, les associations ont dû faire appel à d'autres sources de financement. Parmi celles-ci, notons des dons d'entreprises telles la Spruce Falls, d'autres institutions financières, des corporations, d'autres municipalités, des différents paliers gouvernementaux et de la communauté. Un individu a même indiqué sous cette question «qu'un organisme à but lucratif fait appel à toutes les possibilités dans sa communauté». Les associations ont également puisé leur financement non seulement de dons mais aussi de ventes.

Donc, la Caisse populaire de Kapuskasing semble combler les besoins des associations communautaires puisque leurs représentants et représentantes se disent presque tous satisfaits de l'aide financière contribuée par la caisse, bien qu'elles doivent aussi faire appel à d'autres sources de financement.

\section{Les images de la Caisse véhiculées dans la communauté}

En plus des commentaires positifs mentionnés ci-haut, les répondantes et répondants ont aussi émis des commentaires que nous avons qualifiés de négatifs. En effet, à partir d'une analyse de type qualitatif, nous avons pu dégager les images de la Caisse qui sont véhiculées dans la communauté telles que perçues par les répondantes et répondants. Selon les réponses obtenues, ces images ont trait principalement au fait français, à la qualité des services offerts et à l'engagement dans la communauté. On retrouve aussi tout un ensemble d'images autant positives que négatives. 


\section{Le fait français et la qualité des services}

Seize affirmations par rapport au fait français (dont 6 furent jugées négatives) permettent de conclure que cette caractéristique de la Caisse est relativement importante. La Caisse étant ainsi gérée par des francophones pour des sociétaires majoritairement francophones, il va sans dire que le fait français est une variable importante pour les répondantes et répondants. La qualité des services offerts s'est avérée aussi importante aux yeux des associations participantes. D'après ces dernières, la communauté semble véhiculer des images très positives à l'égard des services offerts par la Caisse. Parmi les commentaires entièrement positifs, on réfere souvent par exemple à la Campagne Noël chez nous, qui prête 1000 \$ sans intérêt pour six mois aux entreprises de la région: «...le programme de Noël pour acheter local chez les marchand locaux ainsi de suite... d'autres institutions financières ont commencé à embarquer dans certains de ces projets-là, euh à cause qui ont vu le positif que ça fait dans communauté...». Or, d'autres ont simplement référé à la bonne qualité des services retrouvés à la Caisse; autrement dit, ils ont révélé la mesure dans laquelle les commentaires entendus dans la communauté s'avèrent positifs: «...la Caisse est toujours là pour nous aider» et "...les caissières sont encore là pi y vont remplir les bordereaux...» font partie des vint-six (26) commentaires positifs qui ont trait à la qualité des services.

\section{L'engagement de la Caisse à sa communauté}

Le thème de l'engagement de la Caisse dans la communauté a été répété le plus souvent. Bien que l'engagement de la Caisse se manifeste de maintes façons, les répondantes et répondants l'ont grandement reconnu lorsqu'elle a prêté 5,4 \$ millions aux membres pour investir dans l'achat de la papetière Spruce Falls Inc. au taux hypothécaire courant plutôt qu'au taux d'un prêt personnel. Par exemple, les «contributions monétaires importantes» et le fait que la Caisse «[connaisse] les besoins de la communauté», c'est-à-dire qu'elle soit «in touch» comme disait un répondant, nous indique l'influence qu'a eue la Caisse lors de cette crise, autant sur ses membres que sur les non membres. D'autres 
remarques ont porté sur les sports, la culture et l'implication de la Caisse auprès des jeunes.

\section{Images positives et négatives}

Nous avons recensé un total de 28 images positives. Nous les avons classées en deux catégories: la première a trait aux images qui «sont bonnes» et à la satisfaction des membres de la Caisse. La seconde porte sur l'apparence physique de la caisse, en raison de commentaires comme «c'est beau» et «je me sens chez nous».

Dix-huit (18) images négatives ont aussi été recensées. Elles ont été regroupées en trois catégories: technique, c'est-à-dire la frustration liée aux pannes du système informatique; l'image de caisse riche (des beaux locaux, à la fine pointe de la technologie, crainte d'érosion des bénéfices); et finalement, le service de crédit, soit des refus de demande et des exigences trop grandes. En somme, les images négatives perçues par les répondantes et répondants démontrent que la Caisse populaire de Kapuskasing possède des côtés plus «faibles» malgré les discours positifs qui sont généralement entendus dans la communauté. Ces images négatives ne concernent pas toujours le personnel qui transige directement avec le public, mais plus spécifiquement, le personnel «derrière les portes cachées» qui traite les demandes d'emprunt. Cela semble représenter une source de mauvaise publicité pour la Caisse et pourrait, à l'extrême, éloigner des sociétaires qui iraient plutôt vers des banques locales.

\section{La perception des répondantes: positive ou négative?}

Les catégories de l'analyse ont démontré que la Caisse populaire de Kapuskasing possède une excellente réputation dans la communauté. Ceci se perçoit dans les nombreux commentaires sur l'adhésion au fait français, sur la qualité des services et sur l'engagement communautaire de la Caisse. Il faut toutefois considérer que la Caisse, c'est une institution comme les autres. 
En d'autres termes, c'est une institution gérée par des personnes qui sont aptes à faire des erreurs. Néanmoins, il est possible d'identifier des conflits parmi ces personnes, augmentant ainsi toujours la possibilité de créer des images négatives au sein de la communauté.

Si les images repérées dans notre recherche donnent de l'importance à la perspective de la communauté, elles ne s'accordent pas nécessairement à la perception qu'ont les répondantes et répondants de la Caisse. En effet, on réalise que la plupart d'entre eux sont plutôt d'accord avec les images positives véhiculées dans la communauté si l'on se fie à des commentaires tels "[puisque les gens] ne [veulent] certainement pas perdre [leur] Caisse» et "[puisque les gens aiment] bien les services». De plus, les répondantes et répondants n'adhèrent pas aux images négatives au sujet de la Caisse.

\section{Conclusion}

Il est évident que les images positives et négatives de notre étude ne tracent pas le portrait réel de toutes les coopératives et les caisses populaires en Ontario français. Bien entendu, des perspectives différentes quant à son avenir font état du virage que subit le mouvement coopératif dans une société de crise comme la nôtre. Bien entendu encore, plusieurs auteurs s'entendent pour dire que c'est une transformation qui abonde dans le sens du «renouveau» qui saura assurer la longue vie du mouvement; cependant le débat n'est toujours pas résolu. En d'autres termes, nous ne pouvons pas nous entendre sur la réelle direction que prend le mouvement coopératif dans la logique sociale et économique actuelle.

Les résultats de notre recherche, à notre avis, ont démontré que la longue vie du mouvement dépend beaucoup des images que se font les personnes de la communauté dans laquelle oeuvre la coopérative ou la caisse populaire. Elle dépend surtout de leur 
satisfaction. La question de «renoncer à ses principes coopératifs» ou non réfere essentiellement aux images que se font les personnes (dans notre cas, les associations) qui font affaire avec l'institution.

Selon nous, le fait d'avoir instauré une mission démontre que la Caisse populaire de Kapuskasing s'est sentie obligée, à la fin des années 1980, de définir et de développer une stratégie pour survivre dans un contexte social de restructuration, adoptant ainsi la perspective tendancielle du «renouveau». Il s'agirait effectivement d'effectuer d'autres recherches afin de permettre aux caisses populaires et aux coopératives de l'Ontario français de réfléchir sur leurs propres pratiques pour se renouveler et durer encore plus longtemps ${ }^{2}$.

\section{Bibliographie}

CAISSE POPULAIRE DE KAPUSKASING (1997). Notre mission [document informatisé].

Http ://www.caissekap.on.ca/mission.htm.

ÉLIE, B. (1997). «Le Mouvement Desjardins de l'an 2000 : des changements obligés», Lévesque, B. (s. la dir. de) Desjardins. Une entreprise et un mouvement?, Québec, Presses de l'Université du Québec, $115-127$.

LABRECQUE, L. (1986). «Le déclin des coopératives de consommation et les mutations d'une culture économique», Recherches sociographiques, vol. 27, no 2, 195-217.

LAFRENIÈRE, G. (1987). La coopération et les Franco-ontariens, Sherbrooke, Institut de recherche et d'enseignement pour les coopératives de l'Université de Sherbrooke, Collection Essais, no11.

LÉVESQUE, B. et M.-C. MALO (1997). «Vue d'ensemble du Mouvement Desjardins en 1996», Lévesque, B. (s. la dir. de). La réingénierie des caisses populaires et d'économie Desjardins, Contexte et étude de cas, CRISES/SAC-UQAM-FC-CSN, 3-27.

\section{Notes}

1. Dupont, Natalie (1998) (s.la dir. de Rachid Bagaoui et Donald Dennie). La perception des associations communautaires de la ville de Kapuskasing à l'égard de l'engagement de la Caisse populaire dans le développement de leur milieu, mémoire de spécialisation. Département de sociologie, Université Laurentienne.

2. J'aimerais remercier particulièrement Bernard Thibodeau, Directeur des communications de la Caisse populaire de Kapuskasing qui, grâce à sa patience et à la lecture attentive de mon mémoire de spécialisation, a pu rédiger une critique sur les résultats de cette étude. 